\title{
HIGHER RANK REPRESENTATIONS AND CAUSALITY
}

R.-K. LOIDE. KORGEMAT JARKU ESITUSED JA POHJUSLIKKUS

P.-К. ЛОИДЕ. ПРЕДСТАВЛЕНИЯ ВЫСШЕГО РАНГА И ПРИЧНННОСТЬ

\section{(Presented by A. Humal)}

In a recent paper [ $\left.{ }^{1}\right]$ there were given two new causal $s=0$ wave equations, using higher rank representations. It was also stated that equations with nilpotent $\beta$-matrices need not be inconsistent. It is well known that the acausality in minimal electromagnetic coupling is necessarily connected with nilpotency of $\beta$-matrices. The examples of causal equations with nilpotent $\beta$-matrices $\left[{ }^{2}\right]$ showed that in this case the nilpotency is not essential but is caused by barnacles (ballast components). Then the equation is equivalent to a simpler equation with diagonalizable $\beta$ matrices which is known to be causal [ $\left.{ }^{3}\right]$.

In this note we demonstrate that the causal $s=0$ equations with higher-rank representations given in ['], are barnacled equations equivalent to the Kemmer-Duffin $s=0$ equation. Therefore these equations are not physically interesting, and indicate that the introduction of higherrank representations is not useful.

Here we treat only the equation with reppresentation $(1,1) \oplus 2(1 / 2,1 / 2) \oplus$ $\oplus(0,0)$, since the other example with representation $(1,0) \oplus 2(1 / 2,1 / 2) \oplus$ $\oplus(0,1) \oplus(0,0)$ may be treated similarly. In the notations of the paper $\left[{ }^{1}\right]$, the equation is

$$
\begin{aligned}
& S_{\mu v}=b_{1}\left\{\partial_{\mu} A_{v}\right\}_{s}+b_{3}\left\{\partial_{\mu} B_{y}\right\}_{s}, \\
& A_{\mu}=\widetilde{b}_{1} \partial^{v} S_{\mu v}+b_{3} \partial_{\mu} \Phi \\
& B_{\mu}=\widetilde{b}_{2} \partial^{v} S_{\mu v}+b_{4} \partial_{\mu} \Phi \\
& \Phi=\widetilde{b}_{3} \partial^{\mu} A_{\mu}+\widetilde{b}_{4} \partial^{\mu} B_{\mu} .
\end{aligned}
$$

Here $\left\{\partial_{\mu} A_{v}\right\}_{S}=1 / 2\left(\partial_{\mu} A_{v}+\partial_{v} A_{\mu}-1 / 2 g_{\mu \nu} \partial^{\rho} A_{\rho}\right)$. There are imposed the following conditions on the coefficients

$$
\begin{gathered}
\widetilde{b}_{1} b_{1}+\widetilde{b}_{2} b_{2}=0, \\
b_{1} b_{3}+b_{2} b_{4}=0 \text { or } \widetilde{b}_{1} \widetilde{b}_{3}+\widetilde{b}_{2} \widetilde{b}_{4}=0, \\
\widetilde{b}_{3} b_{3}+\widetilde{b}_{4} b_{4}<0 .
\end{gathered}
$$

The condition (2) guarantees the nilpotency of $s=1$ block, and leaves only $s=0$ states. The condition (3) gives a unique $s=0$ state, and (4) gives the mass term.

Now we, using (2) and (3), demonstrate the barnacle structure of the equation (1). Depending on the choice of the condition (3), we get different types of barnacles.

1 c a se. $b_{1} b_{3}+b_{2} b_{4}=0$.

Let us introduce new vector fields $C_{\mu}$ and $D_{\mu}$ 


$$
\begin{aligned}
& C_{\mu}=b_{1} A_{\mu}+b_{2} B_{\mu}, \\
& D_{\mu}=\alpha A_{\mu}+\beta B_{\mu},
\end{aligned}
$$

where $\alpha$ and $\beta$ satisfy

$$
d=b_{1} \beta-b_{2} \alpha \neq 0 .
$$

Using (1), (2), (5), and (6), we get a new system for $S_{\mu v}, C_{\mu}, D_{\mu}$, and $\Phi$

$$
\begin{aligned}
& S_{\mu v}=\left\{\partial_{\mu} C_{v}\right\}_{S}, \\
& C_{\mu}=0, \\
& D_{\mu}=\left(\alpha b_{3}+\beta b_{4}\right) \partial_{\mu} \Phi+\left(\alpha \widetilde{b}_{1}+\beta \widetilde{b}_{2}\right) \partial^{v} S_{\mu v}, \\
& \Phi=d^{-1}\left(\widetilde{b_{4}} b_{1}-\widetilde{b}_{3} b_{2}\right) \partial^{\mu} D_{\mu}+d^{-1}\left(\widetilde{b}_{3} \beta-\widetilde{b}_{4} \alpha\right) \partial^{\mu} C_{\mu} .
\end{aligned}
$$

From (7) we can see that $C_{\mu}=0$, and $S_{\mu \nu}=0$. Therefore for $D_{\mu}$ and $\Phi$ we have a separate equation

$$
\begin{aligned}
& D_{\mu}=\left(\alpha b_{3}+\beta b_{4}\right) \partial_{\mu} \Phi, \\
& \Phi=d^{-1}\left(\widetilde{b}_{4} b_{1}-\widetilde{b}_{3} b_{2}\right) \partial^{\mu} D_{\mu} .
\end{aligned}
$$

The equation (8) is equivalent to the well-known Kemmer-Duffin $s=0$ equation.

As we can see, $S_{\mu v}$ and $C_{\mu}$ are the ballast components (barnacles) [ $\left.{ }^{4}\right]$ and are not playing an essential role. Introducing the minimal electromagnetic coupling by substitution $\partial_{\mu} \rightarrow \partial_{\mu}-i e \Phi_{\mu}$, where $\Phi_{\mu}$ is the electromagnetic potential, we do not change the general structure of the equation (7), and, since $C_{\mu}=S_{\mu \nu}=0$, we have the minimally coupled Kemmer-Duffin equation, which is causal. In [ $\left.{ }^{4}\right]$ it is mentioned that the ballast components can play an essential role only in such couplings which change the structure of a given equation, therefore the coupling must be nonminimal.

The coefficients $\alpha$ and $\beta$ are arbitrary except for the condition (6). Since one of the coefficients, $b_{1}$ or $b_{2}$, must be nonzero, the condition (6) may be easily satisfied.

2. c a se. $\widetilde{b}_{1} \widetilde{b}_{3}+\widetilde{b}_{2} \widetilde{b}_{4}=0$.

Now we introduce the following fields $-C_{\mu}$ and $D_{\mu}$

$$
\begin{aligned}
& C_{\mu}=\alpha A_{\mu}+\beta B_{\mu}, \\
& D_{\mu}=\widetilde{b}_{3} A_{\mu}+\widetilde{b}_{4} B_{\mu},
\end{aligned}
$$

where $\alpha$ and $\beta$ satisfy $d=\widetilde{b}_{3} \beta-\widetilde{b}_{4} \alpha \neq 0$.

For $S_{\mu v}, C_{\mu}, D_{\mu}$, and $\Phi$ we get the following system -

$$
\begin{aligned}
& S_{\mu v}=d^{-1}\left(b_{1} \beta-b_{2} \alpha\right)\left\{\partial_{\mu} D_{v}\right\}_{S}+d^{-1}\left(b_{2} \widetilde{b}_{3}-b_{1} \widetilde{b}_{4}\right)\left\{\partial_{\mu} C_{v}\right\}_{S}, \\
& C_{\mu}=\left(b_{1} b_{3}+b_{2} b_{4}\right) \partial_{\mu} \Phi+\left(\alpha \widetilde{b}_{1}+\beta \widetilde{b}_{2}\right) \partial^{v} S_{\mu v}, \\
& D_{\mu}=\left(\widetilde{b}_{3} b_{3}+\widetilde{b}_{4} b_{4}\right) \partial_{\mu} \Phi, \\
& \Phi=\partial^{\mu} D_{\mu} .
\end{aligned}
$$

From (10) we have a different barnacle structure. For $D_{\mu}$ and $\Phi$ we have the Kemmer-Duffin equation. Now, $S_{\mu v}$ and $C_{\mu}$ do not vanish identically. Using the nilpotency condition (2) which eliminates $s=1$, it is possible to verify that $S_{\mu \nu}$ and $C_{\mu}$ are uniquely determined by the 
solution of Kemmer-Duffin equation for $D_{\mu}$ and $\Phi$ (most easily it may be verified choosing $\alpha=b_{1}$ and $\beta=b_{2}$ ). Therefore $S_{\mu v}$ and $C_{\mu}$ are ballast components which do not play an independent role.

The minimal substitution $\partial_{\mu} \rightarrow \partial_{\mu}-i e \Phi_{\mu}$ does not change the general structure of (11), and therefore (10) is causal.

In this note we have shown that the inessential nilpotency (caused by barnacles) does not lead to the acausality. The essential nilpotency, as in $s=0$ equation with the representation $(1,1) \oplus(1 / 2,1 / 2) \oplus(0,0) \oplus$ $\oplus(1,0) \oplus(0,1)$, gives the acausal equation, as has also been demonstrated in $\left.{ }^{1}\right]$. So we can see that the usage of higher rank representations does not help us to deal with causality problems. Higher-rank representations always introduced additional spins which had to be removed by nilpotency. Therefore the higher-rank representations allowed low-spin equations with nilpotent $\beta$-matrices. If the nilpotency is essential, we have acausality; otherwise we have barnacled equations which are equivalent to the simpler equations without higher-rank representations. In conclusion, the introduction of higher-rank representations is not useful.

In our case the barnacles are caused by the repeated representations (two vectors $A_{\mu}$ and $B_{\mu}$ ). As it was first demonstrated in $\left[{ }^{5}\right]$, in the case of repeated representations one has an additional freedom to mix them by unitary transformations and eliminate some of the linkages (see, for example, the Amar-Dozzio equation in $\left.\left[{ }^{4}\right]\right)$. This freedom means that using repeated representations one, in general, gets the barnacled equation (contrary to the proposition [ $\left.{ }^{5}\right]$ stating that we get independent equations).

\section{REFERENCES}

1. C o x. W., J. Phys. A: Math. Gen., 15. № 2, 627-635 (1982).

2. Khalil, M. A. K., Progr. Theor. Phys., 58, № 5, 1538-1554 (1977).

3. A ma r, V., Dozzio. U., Lett. Nuovo Cim., 12, № 17, 659-662 (1975).

4. Lo id e, K., L o ide, R.-K., Preprint F-6, Tartu, 1977.

5. Федоров Ф. И., Плетюхов В. А., Изв, АН БССР, Физ. Матем., 6, 81-88 (1969); 3, 78-83 (1970). 